\title{
Correction to: On the convergence of Mickens' type nonstandard finite difference schemes on Lane-Emden type equations
}

\author{
Amit Kumar Verma ${ }^{1} \cdot$ Sheerin Kayenat ${ }^{1}$
}

Published online: 28 February 2019

(c) Springer Nature Switzerland AG 2019

\section{Correction to: J Math Chem (2018) 56:1667-1706 https://doi.org/10.1007/s10910-018-0880-y}

In the original article, the analytical solution

$$
w(t)=\ln \left(\frac{B+1}{B x^{2}+1}\right),
$$

where $\mathrm{B}=3-2 \sqrt{ } 2$, of Problem 9 (Thermal distribution in the human head [1]) is published incorrectly. That was a typo graphical error. To the best of our knowledge, exact solution of Problem 9 is not known. We replace the term analytical by Table 7 [2] in column 6 of Table 17 and Fig. 25 in the paper. All the data in table and figure were correct in the paper. We just replace the word analytical by Table 7 [2] here.

\begin{tabular}{lllllll}
\hline$t$ & NSFD $(W 1)$ & NSFD $(W 2)$ & NSFD $(W 3)$ & NSFD $(W 4)$ & Table 7 [2] & FD \\
\hline 0 & 0.266263465 & 0.268451865 & 0.269316716 & 0.269692931 & - & 0.269195827 \\
0.1 & 0.266263465 & 0.267495248 & 0.268122301 & 0.268439455 & 0.268756903 & 0.267966449 \\
0.2 & 0.262417516 & 0.263660205 & 0.264292812 & 0.264612699 & 0.26493282 & 0.264131907 \\
0.3 & 0.255974454 & 0.257242592 & 0.257887677 & 0.25821368 & 0.258539792 & 0.257734361 \\
0.4 & 0.246894301 & 0.248207425 & 0.24887461 & 0.249211433 & 0.249548183 & 0.248743821 \\
0.5 & 0.235122416 & 0.236506816 & 0.237208719 & 0.237562447 & 0.237915891 & 0.237099948 \\
0.6 & 0.220590786 & 0.222079284 & 0.222831647 & 0.223209958 & 0.22358771 & 0.22275 \\
0.7 & 0.203215373 & 0.20484866 & 0.205670897 & 0.206083078 & 0.206494486 & 0.205625033 \\
0.8 & 0.182895848 & 0.184722875 & 0.185638095 & 0.186095721 & 0.186552018 & 0.185664293 \\
0.9 & 0.159513476 & 0.161592358 & 0.162628197 & 0.163145114 & 0.163659686 & 0.162760884 \\
1 & 0.132927843 & 0.135328001 & 0.13651715 & 0.137110278 & 0.137698751 & 0.136787351 \\
\hline
\end{tabular}

Note: Comparison of FD, NSFD and Table 7 [2] solutions of Problem 9 [3]

The original article can be found online at https://doi.org/10.1007/s10910-018-0880-y.

Amit Kumar Verma

akverma@iitp.ac.in

Sheerin Kayenat

sheerinkayenat786@gmail.com

1 Department of Mathematics, Indian Institute of Technology Patna, Patna, Bihar 801106, India 


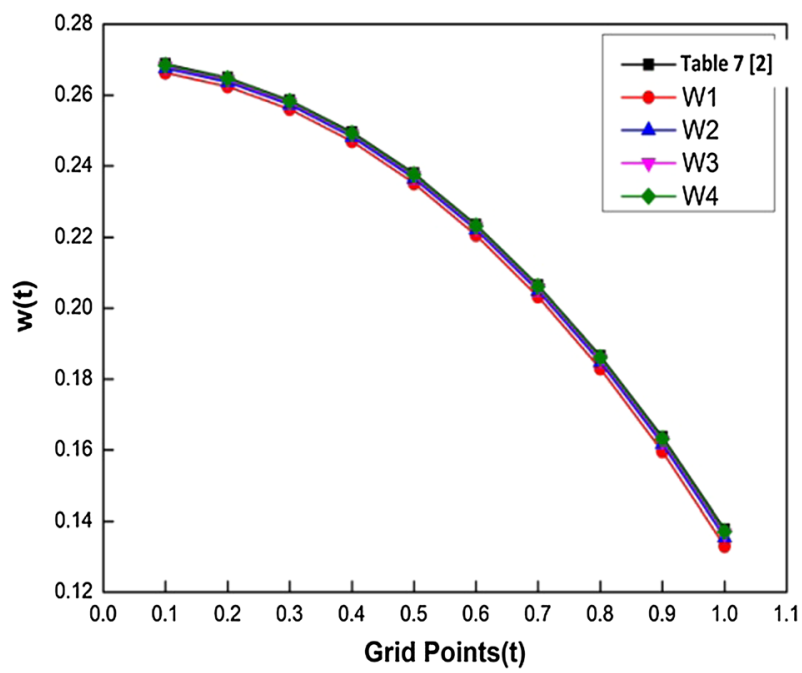

Note: Comparison of NSFD solutions as $h \rightarrow 0$ with solution given in Table 7 [2] for Problem 9 [3]

\section{References}

1. R. Duggan, A. Goodman, Pointwise bounds for a nonlinear heat conduction model of the human head. Bull. Math. Biol. 48(2), 229-236 (1986)

2. M. Singh, A.K. Verma, An effective computational technique for a class of Lane Emden equations. J. Math. Chem. 54, 231-251 (2016)

3. A.K. Verma, S. Kayenat, On the convergence of Mickens' type nonstandard finite difference schemes on Lane Emden type equations. J. Math. Chem. 56(6), 1667-1706 (2018)

Publisher's Note Springer Nature remains neutral with regard to jurisdictional claims in published maps and institutional affiliations. 